\title{
Epithelial-mesenchymal transition in human papillomavirus-positive and -negative oropharyngeal squamous cell carcinoma
}

\author{
HIROMITSU HATAKEYAMA, TAKATSUGU MIZUMACHI, TOMOHIRO SAKASHITA, \\ SATOSHI KANO, AKIHIRO HOMMA and SATOSHI FUKUDA \\ Department of Otolaryngology-Head and Neck Surgery, Hokkaido University \\ Graduate School of Medicine, Kita-ku, Sapporo 060-8638, Japan
}

Received August 7, 2014; Accepted September 1, 2014

DOI: 10.3892/or.2014.3509

\begin{abstract}
High-risk human papillomavirus (HPV) infection is associated with carcinogenesis in oropharyngeal squamous cell carcinoma (OPSCC) and patients with HPV-positive tumors have a significantly favorable prognosis. However, the underlying mechanism of this favorable clinical outcome remains unclear. Epithelial-mesenchymal transition (EMT) causes aggressiveness of cancer cells and we investigated the expression of the EMT markers and analyzed their correlation with HPV status and prognosis in order to examine the treatment response of HPV-positive OPSCCs. A total of 79 patients with OPSCC were examined in the present study. All high-risk HPV infections were determined with the multiplex PCR kit from each formalin-fixed paraffin-embedded (FFPE) sample. We performed immunohistochemical staining for E-cadherin and vimentin. Expression of the markers was graded and we statistically analyzed the correlation between tumor, node, metastasis (TNM) stages and prognosis. Highrisk HPV-positive tumors were detected in 23 cases. The five-year survival rate in HPV-positive and -negative tumors was 82.7 and $48.3 \%$, respectively. High E-cadherin expression rate in HPV-negative samples was $76.7 \%$ and $43.4 \%$ in HPV-positive samples $(\mathrm{p}=0.007)$. Vimentin expression did not show a difference between HPV-positive and -negative tumors. HPV-negative patients presented significantly greater heterogeneity of E-cadherin expression compared to HPV-positive patients $(\mathrm{p}=0.0349)$. HPV-positive OPSCCs originally lost their epithelial cell phenotype compared with HPV-negative tumors. Therefore, the paradoxical favorable prognosis of
\end{abstract}

Correspondence to: Dr Hiromitsu Hatakeyama, Department of Otolaryngology-Head and Neck Surgery, Hokkaido University Graduate School of Medicine, Kita 15, Nishi 7, Kita-ku, Sapporo 060-8638, Japan

E-mail: htkym@med.hokudai.ac.jp

Key words: epithelial-mesenchymal transition, human papillomavirus, oropharyngeal squamous cell carcinoma
HPV-positive OPSCC may be due to the intratumor homogeneity in EMT.

\section{Introduction}

Major risk factors of head and neck squamous cell carcinoma (HNSCC) are environmental factors, typified by tobacco and alcohol abuse, although the carcinogenesis of a number of malignancies, particularly in oropharyngeal sites, is associated with human papillomavirus (HPV) infection. The number of oral and pharyngeal cancers considered to be caused by HPV infection is increasing in the USA (1). Although oncogenic HPV was detected in $25.9 \%$ of all HNSCCs worldwide (2), high-risk HPV type 16 DNA was detected in up to $72 \%$ in oropharyngeal sites and the infection rate of non-smokers with oropharyngeal squamous cell carcinoma (OPSCC) is 15 -fold higher than that of smokers $(3,4)$. Some reports have shown that the prognosis for patients with HPV-induced HNSCC is more favorable than that for patients with HNSCC caused by environmental factors (5-10). In general, the more poorly-differentiated a tumor, the more aggressive its behavior (11) Paradoxically, most HPV-positive HNSCCs are histologically graded as poorly differentiated in spite of their favorable clinical outcomes.

E-cadherin is a cell-cell adhesion molecule and a representative molecular marker of epithelial cells. The loss of E-cadherin expression is a distinctive event in epithelial-mesenchymal transition (EMT), which is a process involving a high degree of cellular plasticity and a large number of distinct genetic and epigenetic alterations, as well as the conversion of differentiated epithelial cells into poorly differentiated, migratory and invasive mesenchymal cells (12-14). E-cadherin is often heterogeneously expressed in HNSCCs. In oral SCCs, E-cadherin is highly expressed at the centre of the tumor, with the expression gradually decreasing toward the invasive front $(15,16)$. Vimentin is highly expressed in mesenchymal cells and is generally used as the molecular marker to identify cancer cells undergoing EMT (17).

In the present study, we investigated the expression of the EMT markers and analyzed their correlation with HPV status and prognosis in order to clarify the reason for the paradoxical treatment response of HPV-positive OPSCCs. 


\section{Patients and methods}

The study group comprised 79 Japanese patients who were diagnosed with OPSCC and treated at Hokkaido University Hospital, Japan, between 1998 and 2010. The main clinical characteristics of the patients are shown in Table I. The subjects included 69 men and 10 women with a mean age of 62.9 years (range, 40-85 years). Demographic and clinicopathological data, including age, gender, smoking history, tumor stage and clinical outcomes, were obtained from the patient charts. All patients were treated with surgery, radiotherapy or chemoradiotherapy. Sixteen patients were treated with surgery, 15 were treated using radiotherapy at a total dose of 65-70 Gy, with 50 Gy received concurrently with chemoradiotherapy (39 of these were treated with concomitant platin-based chemoradiotherapy and 11 were treated with concomitant docetaxel radiotherapy). Median follow-up time was 54 months for surviving patients, and 43 months when deceased patients were included. The present study was approved by the Institutional Review Board of Hokkaido University Hospital (013-0271).

HPV typing by multiplex polymerase chain reaction (PCR). DNA was extracted from five $10-\mu \mathrm{m}$ sections of paraffinembedded pre-treatment tissue obtained from biopsies. Overall, 16 different HPV genotypes (HPV-6, HPV-11, HPV-16, HPV-18, HPV-30, HPV-31, HPV-33, HPV-35, HPV-39, HPV-45, HPV-51, HPV-52, HPV-56, HPV-58, HPV-59 and HPV-66) were detected by multiplex PCR. Mutiplex PCR was conducted as described by Nishiwaki et al (18). In brief, HPV-genotype-specific primers were designed on the basis of multiplex-sequence alignments. PCRs were performed with a multiplex PCR kit (Qiagen Inc., Valencia, CA, USA), according to the manufacturer's instructions, with minor modifications. The HPV-genotypes in the samples were identified by amplicon size.

Statistical analysis. Factors associated with HPV status including gender; age; tumor subsites; tumor, node, metastasis (TNM) status; primary tumor size and involvement of lymph nodes were analyzed by cross-tabulations using the two-tailed Fisher's exact test. Statistical significance was set at $\mathrm{p}<0.05$. Overall survival curves were calculated using the Kaplan-Meier method. Survival was calculated from the date of treatment initiation until either mortality or the last date on which the patient was known to be alive. The statistical significance of differences between survival times was determined by the log-rank test in univariate analyses at a significance level of $\mathrm{p}<0.05$.

Immunohistochemistry. Paraffin-embedded tumor specimens from the primary sites were obtained from all the patients. These specimens were cut into 4-mm sections. They were then deparaffinized in xylene, dehydrated through graded alcohol and placed in $0.1 \%$ hydrogen peroxide to quench any endogenous peroxidase activity. Following 3-times of microwave pretreatment in a citrate buffer $(\mathrm{pH} 6.0)$ for $5 \mathrm{~min}$ at $750 \mathrm{~W}$, these sections were treated with a $10 \%$ normal rabbit serum for $30 \mathrm{~min}$ to prevent non-specific binding of the antibody. The slides were then incubated with the specific monoclonal antibody to human E-cadherin or vimentin in a humid chamber
Table I. Clinicopathological features of all 79 patients.

\begin{tabular}{|c|c|c|c|c|}
\hline & $\begin{array}{c}\text { Patients } \\
\mathrm{N}=79\end{array}$ & $\begin{array}{l}\text { HPV-pos } \\
\mathrm{N}=23\end{array}$ & $\begin{array}{l}\text { HPV-neg } \\
\text { N=56 }\end{array}$ & P-value \\
\hline Gender & & & & NS \\
\hline Male & 69 & 18 & 51 & \\
\hline Female & 10 & 5 & 5 & \\
\hline $\begin{array}{l}\text { Age, years } \\
\text { (median, 63) }\end{array}$ & & & & NS \\
\hline$<63$ & 34 & 13 & 21 & \\
\hline$\geq 63$ & 45 & 10 & 35 & \\
\hline Grade & & & & $0.0074^{\mathrm{a}}$ \\
\hline Well & 14 & 0 & 14 & \\
\hline Moderate & 49 & 17 & 32 & \\
\hline Poor & 15 & 6 & 9 & \\
\hline Unknown & 1 & 0 & 1 & \\
\hline TNM stage & & & & $0.0162^{\mathrm{b}}$ \\
\hline I & 7 & 1 & 6 & \\
\hline II & 11 & 0 & 11 & \\
\hline III & 22 & 7 & 15 & \\
\hline IV & 39 & 15 & 24 & \\
\hline $\begin{array}{l}\text { Primary tumor } \\
\text { (T class) }\end{array}$ & & & & $\mathrm{NS}^{\mathrm{c}}$ \\
\hline 1 & 11 & 3 & 8 & \\
\hline 2 & 31 & 9 & 22 & \\
\hline 3 & 23 & 8 & 15 & \\
\hline 4 & 11 & 1 & 10 & \\
\hline $\mathrm{X}$ & 4 & 2 & 2 & \\
\hline $\begin{array}{l}\text { Lymph nodes } \\
\text { ( } \mathrm{N} \text { class) }\end{array}$ & & & & $0.0212^{\mathrm{d}}$ \\
\hline 0 & 31 & 4 & 27 & \\
\hline 1 & 12 & 4 & 8 & \\
\hline $2 a$ & 2 & 1 & 1 & \\
\hline $2 b$ & 21 & 11 & 10 & \\
\hline $2 c$ & 10 & 2 & 8 & \\
\hline 3 & 4 & 1 & 3 & \\
\hline $\begin{array}{l}\text { Overall survival } \\
\text { at } 5 \text { years }(\%)\end{array}$ & 60.2 & 82.7 & 48.3 & 0.0013 \\
\hline
\end{tabular}

NS, not significant; pos, positive; neg, negative. ${ }^{\text {a}}$ Well vs. moderate and poorly differentiated; ${ }^{\mathrm{b}}$ stage I and II vs. stage III and IV; ${ }^{\mathrm{c}} \mathrm{T} 1-2$ vs. T3-4, ${ }^{\mathrm{N}} \mathrm{N} 0 \mathrm{vs.} \mathrm{N1-3.} \mathrm{HPV,} \mathrm{human} \mathrm{papillomavirus.}$

at $37.8^{\circ} \mathrm{C}$ overnight. The sections were then incubated with a biotin-labeled rabbit anti-mouse secondary antibody [Histofine SAB-PO (M) kit; Nichirei, Tokyo, Japan] for $30 \mathrm{~min}$ at $37.8^{\circ} \mathrm{C}$, followed by reaction with a streptavidin-biotin horseradish peroxidase complex. We observed the reaction products by immersing the slides in a freshly prepared diaminobenzidine solution for $10 \mathrm{~min}$ and counterstaining them with hematoxylin before dehydration and mounting. We determined the percentage of vimentin tumor cells by counting the number of brown-stained tumor cells in the most highly stained area of each slide. The vimentin index was calculated from the ratio of 


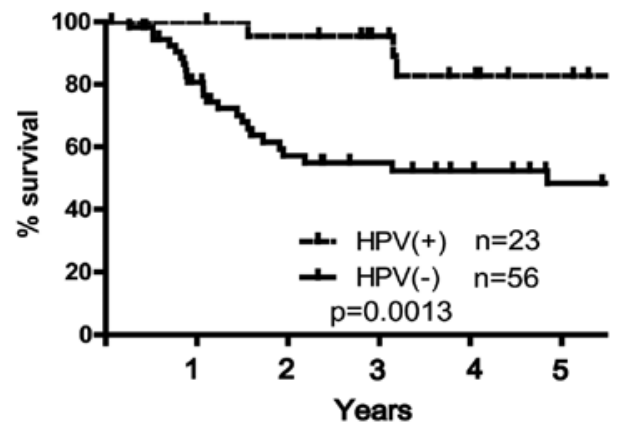

Figure 1. Overall survival according to HPV status in 79 patients with OPSCC. HPV, human papillomavirus; OPSCC, oropharyngeal squamous cell carcinoma.

the number of positively stained tumor cells to the total number of tumor cells per section. Vimentin expression in mesenchymal cells other than tumor cells (blood vessels, stromal tissues) was excluded by observation of hematoxylin and eosin (H\&E)-stained slides. We determined that tumor tissue with a vimentin index $\geq 20 \%$ was positive. For E-cadherin, we graded the staining intensity as follows: 0 , negative; 1 , weak; 2 , moderate; and 3, strong. The proportion of positive cells was categorized as follows: $0,<1 \% ; 1,2-40 \% ; 2,40-80 \% ; 3,>80 \%$. The two scores were then multiplied and the final immunostaining score was determined; a score of 0 was regarded as negative, 2 and 3 as weakly positive, 3 and 4 as moderately positive, and 6-9 as strongly positive. This scoring system was based on one described in a previous report (16). The heterogeneity of E-cadherin expression was estimated by the presence of strongly stained cancer cell nests adjacent to negative or weakly stained cancer cell nests. Furthermore, we repeated the immunostaining thrice and confirmed the results to avoid counting irregular staining. All specimens were examined by two observers (H.H. and T.M.)

\section{Results}

\section{Patients and treatment}

HPV status and clinicopathological features. In total, 23 of the 79 OPSCC tissue samples were found to be HPV-positive. Among them, 20 patients (87\%) were positive for HPV-16, two $(8.7 \%)$ were positive for HPV-18 and one (4.3\%) was positive for HPV-58. No patient presented with dual infections.

The correlations between HPV status and clinicopathological features are shown in Table I. Gender, age and T class were not correlated with HPV status. HPV-positive patients presented significantly more often with lymph-node metastasis compared to HPV-negative patients $(\mathrm{p}=0.0212)$. The HPV-positive OPSCCs were significantly more often histopathologically graded as moderately or poorly differentiated $(\mathrm{p}=0.007)$. Kaplan-Meier analysis showed that HPV-positive patients had an improved 5-year overall survival rate compared with HPV-negative patients ( 82.7 vs. $48.3 \%$, respectively; $\mathrm{p}=0.0013, \log$-rank test; Fig. 1).

Expression of E-cadherin and vimentin in OPSCC and its correlation to clinicopathological features. Immunohistochemical staining for E-cadherin and vimentin was performed in 79 OPSCC tissue samples. Intensity of the staining was confirmed by staining the normal pharyngeal epithelium. E-cadherin expression was observed mainly at the basal and spinosum layers and gradually disappeared in cells that had undergone keratinization (Fig. 2A). Representative weak and strong staining for E-cadherin is shown in
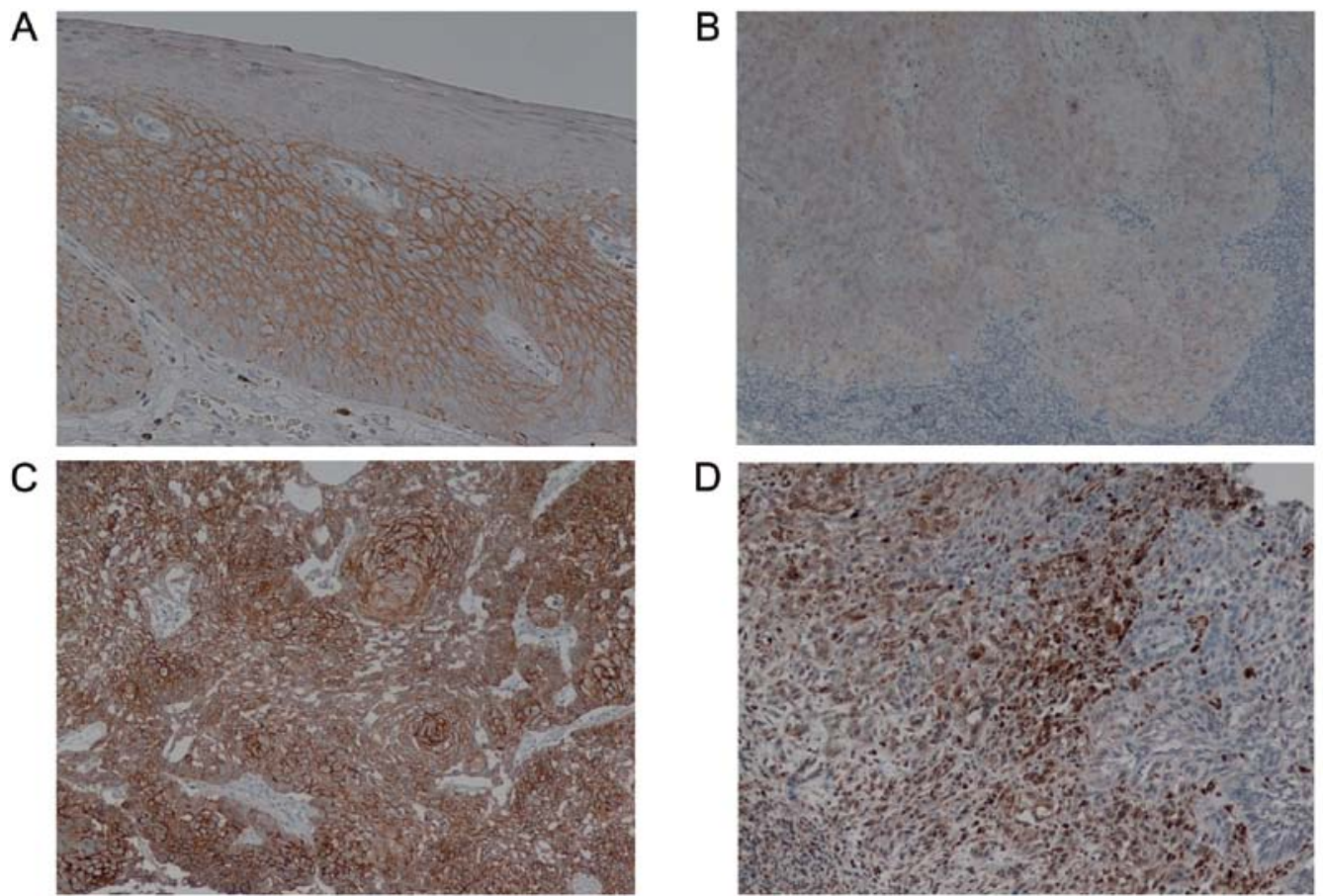

Figure 2. Immunohistochemical staining for E-cadherin and vimentin in 79 OPSCC tissue samples. (A) E-cadherin expression in normal epithelium. (B) Weak E-cadherin expression in OPSCC. (C) Strong E-cadherin expression in OPSCC. (D) Positive vimentin expression in OPSCC. OPSCC, oropharyngeal squamous cell carcinoma. 
Table II. E-cadherin and vimentin expression.

A, E-cadherin expression

\begin{tabular}{lccccc}
\hline & & \multicolumn{4}{c}{ E-cadherin expression } \\
\cline { 3 - 6 } Status & $\mathrm{n}$ & Negative & Weak & Moderate & Strong \\
\hline HPV status & & & & 0.007 \\
Positive & 23 & 3 & 10 & 5 & 5 \\
Negative & 56 & 6 & 7 & 14 & 29 \\
\hline
\end{tabular}

B, Vimentin expression

\begin{tabular}{|c|c|c|c|c|}
\hline \multirow[b]{2}{*}{ Status } & \multirow[b]{2}{*}{$\mathrm{n}$} & \multicolumn{2}{|c|}{ Vimentin expression } & \multirow[b]{2}{*}{ P-value } \\
\hline & & Negative & Positive & \\
\hline HPV status & & & & NS \\
\hline Positive & 23 & 15 & 8 & \\
\hline Negative & 56 & 41 & 15 & \\
\hline
\end{tabular}

NS, not significant. All statistical analyses were carried out as negative and weak vs. moderate and strong. HPV, human papillomavirus.
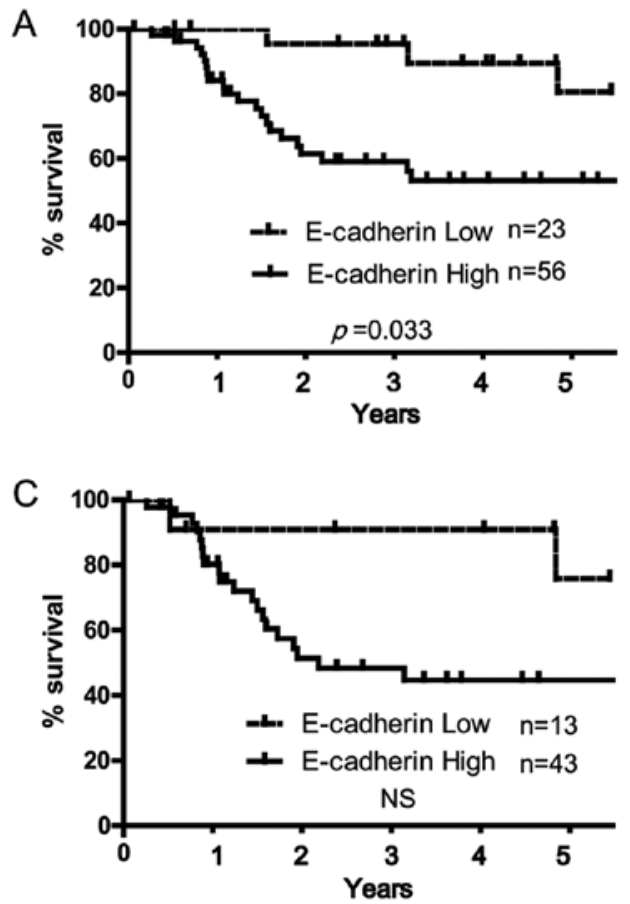

Fig. 2B and C. Strong and moderate E-cadherin staining was observed in 43/56 HPV-positive tumors (76.7\%) and in 10/23 (43.4\%) HPV-negative tumors $(\mathrm{p}=0.007)$ (Table IIA). High and low E-cadherin expression significantly correlated with all 5-year survival rates in 79 OSCC patients $(81.8 \%$ in high E-cadherin expression vs. $53.2 \%$ in low, respectively; $p=0.032$, log-rank test; Fig. 3A). However, E-cadherin expression did not significantly correlate with 5-year survival rate either in HPV-positive or -negative OSCC patients (Fig. 3B and C).

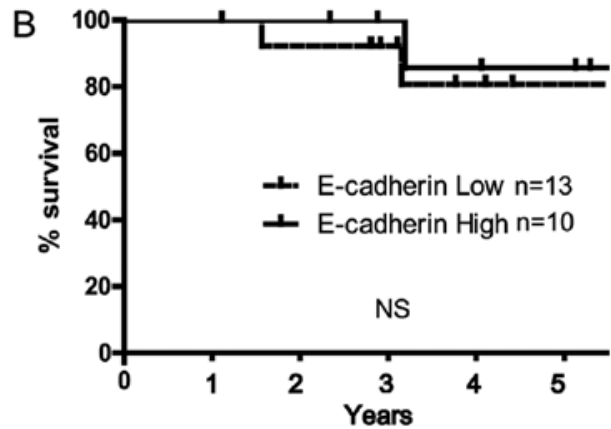

Figure 3. Overall survival according to E-cadherin expression. Kaplan-Meier curves for 5-year overall survival stratified by E-cadherin expression of 79 patients with OPSCC (A), 23 patients with HPV-positive OPSCC (B) and 56 patients with HPV-negative OPSCC (C). OPSCC, oropharyngeal squamous cell carcinoma; HPV, human papillomavirus.

Positive rate of vimentin expression was $29.1 \%$ in all OSCC patients. A typical image of positive vimentin expression is shown in Fig. 2D. Vimentin expression rate was not associated with any clinicopathological features and HPV status (Table IIB). Although we expected vimentin expression to play a counterpart of E-cadherin as the representative marker of mesenchymal phenotype, there was no inverse correlation between E-cadherin and vimentin expression. Therefore, there was no statistical difference in the 5-year survival rates of the 

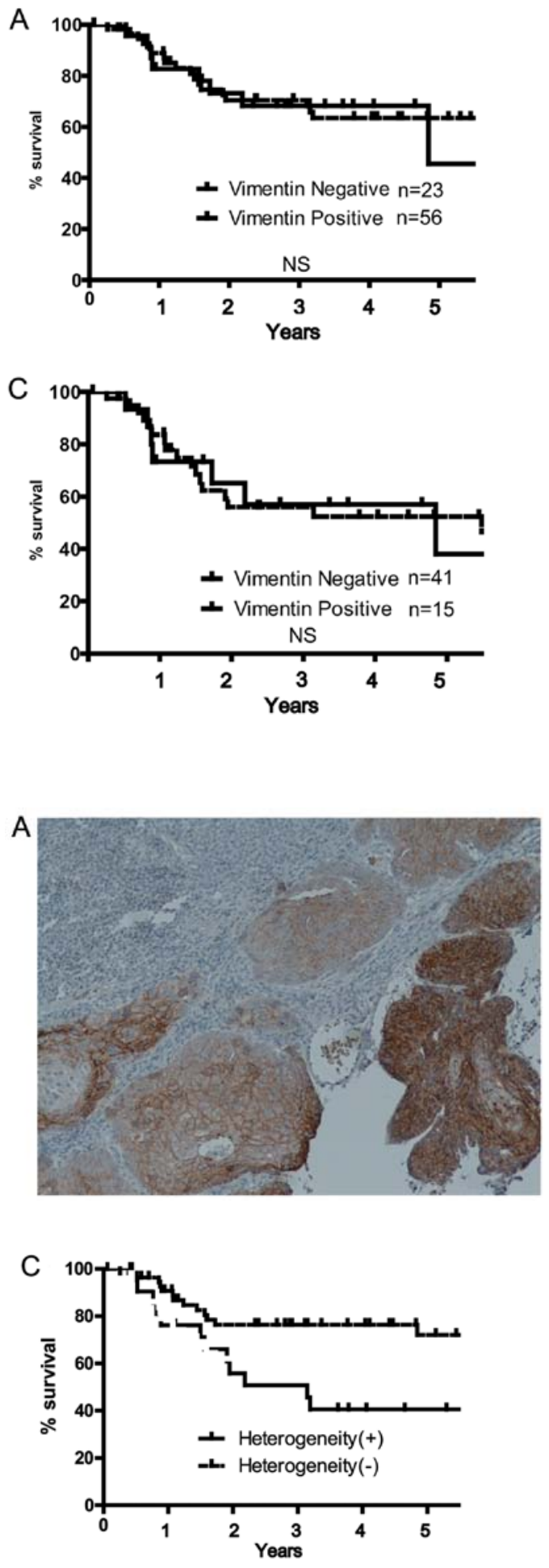

patients with HPV-positive and -negative OSCC with regard to vimentin expression (Fig. 4A-C).

Heterogeneity of E-cadherin expression was found in 23 samples. Typical heterogeneous E-cadherin expression is shown in Fig. 5. HPV-negative patients presented significantly greater heterogeneity of E-cadherin expression compared to HPV-positive patients ( $\mathrm{p}=0.0349)$ (Table III). Fig. 5A and B shows that patients with a heterogeneous expression of E-cadherin had significantly higher 5-year survival rates compared to the patients with homogeneous E-cadherin expression (Fig. 5C).

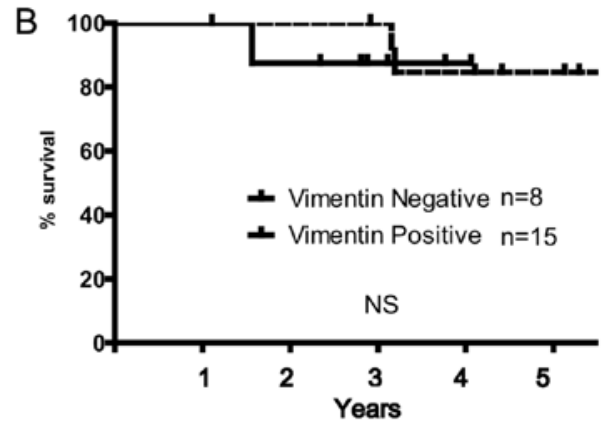

Figure 4. Overall survival according to vimentin expression. Kaplan-Meier curves for 5-year overall survival stratified by vimentin expression of 79 patients with OPSCC (A), 23 patients with HPV-positive OPSCC (B) and 56 patients with HPV-negative OPSCC $(\mathrm{C})$. OPSCC, oropharyngeal squamous cell carcinoma; HPV, human papillomavirus.

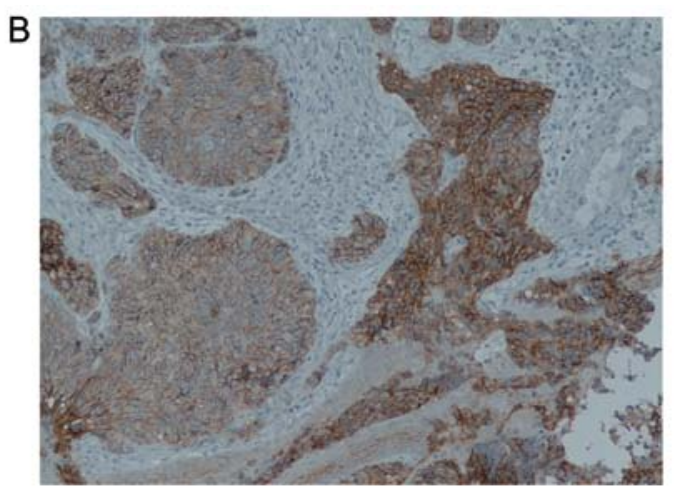

Figure 5. Heterogeneity of E-cadherin expression. (A and B) Typical heterogeneous E-cadherin expression in OPSCC. (C) Kaplan-Meier curves for 5-year overall survival stratified by E-cadherin heterogeneous expression of 79 patients with OPSCC. OPSCC, oropharyngeal squamous cell carcinoma.

Table III. Heterogeneity of E-cadherin expression.

\begin{tabular}{lccc}
\hline & \multicolumn{2}{c}{ E-cadherin expression } & \\
\cline { 2 - 3 } Status & Heterogeneous & Homogeneous & P-value \\
\hline HPV status & 3 & 20 & 0.0349 \\
Positive & 20 & 36 & \\
Negative & 20 & & \\
\hline
\end{tabular}

HPV, human papillomavirus. 


\section{Discussion}

The phenotype of HPV-positive HNSCC is completely different from that of HPV-negative HNSCC, both clinically and pathologically (11). Several molecular studies have also indicated that HPV-positive tumors do not show the same frequent oncogenic molecular alternations which characterize HPV-negative HNSCC, such as mutations of p53, mutations and deletions in $16^{\mathrm{INK} 4 \mathrm{a}}$, overexpression of cyclin D1 or increased EGFR copy numbers (19-24).

Loss of E-cadherin expression significantly correlated with an increased risk of distant metastasis in $\operatorname{HNSCC}(15,25)$. Meta-analysis of existing studies also showed a correlation between low E-cadherin expression and poor prognosis of HNSCC (16), although some studies showed no correlation between these factors (26). Our results, which reveal a more favorable prognosis in patients with OPSCC with low E-cadherin expression, showed an opposite outcome from previous studies as our investigation was limited to oropharynx sites and E-cadherin expression is significantly lower in HPV-positive OPSCC than in HPV-negative cases. The HPV-positive rate in OPSCC is higher than that in other sites, thus they should not be analyzed as the same type of tumor (27). Although most previous reports on E-cadherin expression in HNSCC included all HNSCC sites, it is better to distinguish between HPV-positive and -negative cases for analysis if they include OPSCC. The most frequent subtype of tonsillar HPV detected in the present study was HPV16, a high-risk subtype of HPV for OPSCC, which was in agreement with the results of previous studies $(28,29)$. Several reports have shown that HPV16-E6/E7 regulate E-cadherin expression. E-cadherin expression in the epithelium was shown to be reduced during HPV16 infection, which is associated with the depletion of Langerhans cells at the site of the infection $(30,31)$. A recent study showed that HPV16-E6/E7 regulated E-cadherin expression and induced ZEB1, an EMT-activating transcriptional factor (32). These studies suggested HPV infection induces EMT, yet they did not demonstrate the reason for the favorable clinical outcome in cases of HPV-positive HNSCC.

Intratumor heterogeneity has been shown genetically and is associated with the prognosis and drug resistance in patients with cancer (33). In HNSCC, Mroz and Rocco (34) developed a new system for measuring genetic heterogeneity using next-generation sequencing data, and showed that high genetic heterogeneity is associated with tumor progression and poor treatment outcome. Furthermore, they reported that HPV-positive tumor tissues showed significantly greater intratumor homogeneity than did HPV-negative tissues, which is in agreement with our data $(34,35)$. From these results, a favorable clinical response in cases of HPV-positive HNSCC can be reasoned by their low heterogeneity. No quantitative evaluation of heterogeneity based on EMT has been established. In general, HNSCC biopsy samples are small and we see merely the tip of the whole tumor. Accurate analysis of heterogeneity requires whole tumor specimens both from the primary and the metastasis sites. We expected vimentin expression to play a role as a counterpart to E-cadherin, but vimentin expression was low, even in E-cadherin-negative cancer tissues. The vimentin expression rate is inherently low in SCC; it may not be an appropriate marker in opposition to
E-cadherin. N-cadherin and fibronectin have also been used as markers for mesenchymal cells, yet the molecular expression of these compounds is not sufficiently sensitive to reflect mesenchymal phenotype in HNSCC $(15,36)$.

The results of the present study indicate that HPV-positive tumors tend to lose an epithelial phenotype and were found to be homogeneous based on EMT analysis. This may be the reason for the paradoxical favorable clinical outcome in HPV-positive patients. Future development of an estimation system for intratumor heterogeneity based on EMT may have important applications to clinical decision-making and tailormade therapy in cases of HNSCC.

\section{References}

1. Jemal A, Siegel R, Ward E, et al: Cancer statistics, 2008. CA Cancer J Clin 58: 71-96, 2008.

2. Kreimer AR, Clifford GM, Boyle P and Franceschi S: Human papillomavirus types in head and neck squamous cell carcinomas worldwide: a systematic review. Cancer Epidemiol Biomarkers Prev 14: 467-475, 2005.

3. Lindel K, Beer KT, Laissue J, Greiner RH and Aebersold DM: Human papillomavirus positive squamous cell carcinoma of the oropharynx: a radiosensitive subgroup of head and neck carcinoma. Cancer 92: 805-813, 2001.

4. D'Souza G, Kreimer AR, Viscidi R, et al: Case-control study of human papillomavirus and oropharyngeal cancer. $\mathrm{N}$ Engl $\mathrm{J}$ Med 356: 1944-1956, 2007.

5. Kumar B, Cordell KG, Lee JS, et al: EGFR, p16, HPV titer, Bcl-xL and p53, sex, and smoking as indicators of response to therapy and survival in oropharyngeal cancer. J Clin Oncol 26: 3128-3137, 2008.

6. Worden FP, Kumar B, Lee JS, et al: Chemoselection as a strategy for organ preservation in advanced oropharynx cancer: response and survival positively associated with HPV16 copy number. J Clin Oncol 26: 3138-3146, 2008.

7. Licitra L, Perrone F, Bossi P, et al: High-risk human papillomavirus affects prognosis in patients with surgically treated oropharyngeal squamous cell carcinoma. J Clin Oncol 24: 5630-5636, 2006.

8. Weinberger PM, Yu Z, Haffty BG, et al: Molecular classification identifies a subset of human papillomavirus-associated oropharyngeal cancers with favorable prognosis. J Clin Oncol 24: 736-747, 2006.

9. Fakhry C, Westra WH, Li S, et al: Improved survival of patients with human papillomavirus-positive head and neck squamous cell carcinoma in a prospective clinical trial. J Natl Cancer Inst 100: 261-269, 2008.

10. Mizumachi T, Kano S, Sakashita T, et al: Improved survival of Japanese patients with human papillomavirus-positive oropharyngeal squamous cell carcinoma. Int J Clin Oncol 18: 824-828, 2013.

11. Westra WH: The morphologic profile of HPV-related head and neck squamous carcinoma: implications for diagnosis, prognosis, and clinical management. Head Neck Pathol 6 (Suppl 1): S48-S54, 2012.

12. Yilmaz M and Christofori G: EMT, the cytoskeleton, and cancer cell invasion. Cancer Metastasis Rev 28: 15-33, 2009.

13. Kalluri R: EMT: when epithelial cells decide to become mesenchymal-like cells. J Clin Invest 119: 1417-1419, 2009.

14. Kalluri R and Weinberg RA: The basics of epithelial-mesenchymal transition. J Clin Invest 119: 1420-1428, 2009.

15. Hashimoto T, Soeno Y, Maeda G, et al: Progression of oral squamous cell carcinoma accompanied with reduced E-cadherin expression but not cadherin switch. PLoS One 7: e47899, 2012.

16. Zhao Z, Ge J, Sun Y, et al: Is E-cadherin immunoexpression a prognostic factor for head and neck squamous cell carcinoma (HNSCC)? A systematic review and meta-analysis. Oral Oncol 48: 761-767, 2012.

17. Zeisberg $M$ and Neilson EG: Biomarkers for epithelial-mesenchymal transitions. J Clin Invest 119: 1429-1437, 2009.

18. Nishiwaki M, Yamamoto T, Tone S, et al: Genotyping of human papillomaviruses by a novel one-step typing method with multiplex PCR and clinical applications. J Clin Microbiol 46: 1161-1168, 2008 
19. Olshan AF, Weissler MC, Pei H, et al: Alterations of the p16 gene in head and neck cancer: frequency and association with p53, PRAD-1 and HPV. Oncogene 14: 811-818, 1997.

20. Braakhuis BJ, Snijders PJ, Keune WJ, et al: Genetic patterns in head and neck cancers that contain or lack transcriptionally active human papillomavirus. J Natl Cancer Inst 96: 998-1006, 2004.

21. Haraf DJ, Nodzenski E, Brachman D, et al: Human papilloma virus and p53 in head and neck cancer: clinical correlates and survival. Clin Cancer Res 2: 755-762, 1996.

22. Perrone F, Suardi S, Pastore E, et al: Molecular and cytogenetic subgroups of oropharyngeal squamous cell carcinoma. Clin Cancer Res 12: 6643-6651, 2006.

23. Kessis TD, Slebos RJ, Nelson WG, et al: Human papillomavirus 16 E6 expression disrupts the p53-mediated cellular response to DNA damage. Proc Natl Acad Sci USA 90: 3988-3992, 1993.

24. Koch WM, Lango M, Sewell D, Zahurak M and Sidransky D: Head and neck cancer in nonsmokers: a distinct clinical and molecular entity. Laryngoscope 109: 1544-1551, 1999.

25. Nijkamp MM, Span PN, Hoogsteen IJ, van der Kogel AJ, Kaanders JH and Bussink J: Expression of E-cadherin and vimentin correlates with metastasis formation in head and neck squamous cell carcinoma patients. Radiother Oncol 99: 344-348, 2011.

26. Andrews NA, Jones AS, Helliwell TR and Kinsella AR: Expression of the E-cadherin-catenin cell adhesion complex in primary squamous cell carcinomas of the head and neck and their nodal metastases. Br J Cancer 75: 1474-1480, 1997.

27. Baumann JL, Cohen S, Evjen AN, et al: Human papillomavirus in early laryngeal carcinoma. Laryngoscope 119: 1531-1537, 2009.
28. Kreimer AR, Alberg AJ, Daniel R, et al: Oral human papillomavirus infection in adults is associated with sexual behavior and HIV serostatus. J Infect Dis 189: 686-698, 2004.

29. Syrjänen S: Human papillomavirus (HPV) in head and neck cancer. J Clin Virol 32 (Suppl 1): S59-S66, 2005.

30. Vessey CJ, Wilding J, Folarin N, et al: Altered expression and function of E-cadherin in cervical intraepithelial neoplasia and invasive squamous cell carcinoma. J Pathol 176: 151-159, 1995.

31. Matthews K, Leong CM, Baxter L, et al: Depletion of Langerhans cells in human papillomavirus type 16-infected skin is associated with E6-mediated down regulation of E-cadherin. J Virol 77: 8378-8385, 2003.

32. Jung YS, Kato I and Kim HR: A novel function of HPV16-E6/ E7 in epithelial-mesenchymal transition. Biochem Biophys Res Commun 435: 339-344, 2013.

33. Gerlinger M, Rowan AJ, Horswell S, et al: Intratumor heterogeneity and branched evolution revealed by multiregion sequencing. N Engl J Med 366: 883-892, 2012.

34. Mroz EA and Rocco JW: MATH, a novel measure of intratumor genetic heterogeneity, is high in poor-outcome classes of head and neck squamous cell carcinoma. Oral Oncol 49: 211-215, 2013.

35. Mroz EA and Rocco JW: Gene expression analysis as a tool in early-stage oral cancer management. J Clin Oncol 30: 4053-4055, 2012.

36. Thiery JP, Acloque H, Huang RY and Nieto MA: Epithelialmesenchymal transitions in development and disease. Cell 139: 871-890, 2009. 